\title{
Féeries
}

Études sur le conte merveilleux, XVII $-\mathrm{XIX}{ }^{\mathrm{e}}$ siècle

\section{Les contes, un laboratoire des sens : augmentation sensorielle, polysensorialité et synesthésies}

Tales as a laboratory of the senses: sensory increase, polysensoriality and synaesthesia

\section{Aurélia Gaillard}

\section{OpenEdition}

\section{Journals}

Édition électronique

URL : http://journals.openedition.org/feeries/1276

DOI : 10.4000/feeries. 1276

ISSN : $1957-7753$

Éditeur

UGA Éditions/Université Grenoble Alpes

Édition imprimée

ISBN : 978-2-37747-075-4

ISSN : 1766-2842

\section{Référence électronique}

Aurélia Gaillard, «Les contes, un laboratoire des sens : augmentation sensorielle, polysensorialité et synesthésies », Féeries [En ligne], 15 | 2018, mis en ligne le 22 janvier 2019, consulté le 08 septembre 2020. URL : http://journals.openedition.org/feeries/1276 ; DOI : https://doi.org/10.4000/feeries.1276

Ce document a été généré automatiquement le 8 septembre 2020

(c) Féeries 


\title{
Les contes, un laboratoire des sens : augmentation sensorielle, polysensorialité et synesthésies
}

\author{
Tales as a laboratory of the senses: sensory increase, polysensoriality and \\ synaesthesia
}

Aurélia Gaillard

On connaît bien sûr chez Montesquieu, dans un conte inséré des Lettres persanes, l'épisode de la saturation des sens qu'éprouve Anaïs dans le sérail inversé d'Ibrahim $-\mathrm{y}$ compris jusqu'à la violence et la sidération ${ }^{1}$. L'association des plaisirs de la vue (belle architecture, beaux corps), avec ceux de la musique, du toucher délicat, des parfums enivrants exhalés par de précieuses cassolettes, voire de ragoûts raffinés, est une topique récurrente des palais et des sérails des contes des $\mathrm{XVII}^{\mathrm{e}}$ et $\mathrm{XVIII}{ }^{\mathrm{e}}$ siècles; même si celle-ci a bien entendu une dimension et un enjeu tout à fait exceptionnels dans le conte d'Anaïs et Ibrahim². À des degrés divers, avec plus ou moins d'inventivité, nombre de palais, de sérails ou encore de temples se présentent comme des espaces d'intensification sensorielle et de polysensorialité. Divers dispositifs optiques, de diffraction, d'anamorphose, de mobilité, tentent parfois aussi de rendre sensible pour le lecteur l'effet synesthésique de la correspondance entre plusieurs sens, souvent l'ouïe et la vue. Dans un conte étiologique anonyme, narrant l'origine des poupées, Le Navire volant, on est ainsi conduit dans le salon polysensoriel d'un palais de diamants, où la combinaison des deux motifs des tableaux et des instruments de musique animés, dirigés de surcroît par l'action des spectateurs eux-mêmes, procurent un effet d'harmonie de type synesthésique. Au «miracle de la vue» se joignait «celui de l'ouïe ", écrit le conteur. Miracle de la vue, donc, provoqué par des tableaux mouvants : «Quant aux murailles de ce beau salon, elles représentaient tour à tour, et suivant la volonté de celles qui les considéraient, toutes les personnes qui habitent l'univers.» Miracle de l'ouïe, quant à lui, induit par une musique sensible : « Pour mettre en œuvre ces instruments, il suffisait d'ordonner à celui dont on aimait le mieux le son, de rapporter les paroles que proféraient les personnes pour lesquelles on avait de la 
curiosité ; l'instrument obéissait aussitôt, avec beaucoup de fidélité et de mélodie ${ }^{3}$. $\mathrm{Ce}$ type de dispositif, optique et musical tout à la fois, est sans doute le plus courant, on le retrouve par exemple dans un épisode du conte anonyme de 1750, Le Prince Ananas et la Princesse Moustelle. La Princesse Moustelle, dont le défaut est la coquetterie, se rend comme toutes «les jeunes personnes» de la Cour dans un temple appelé « le temple de la curiosité ». Or, celui-ci a pour caractéristique non seulement de faire se correspondre les deux sens de la vue et de l'ouïe, mais aussi de les intensifier grâce à une architecture sonore et une saturation de tableaux sur tous les murs :

La déesse [du temple] est représentée couverte d'yeux et d'oreilles; son temple est sonore, et tout ouvert; le plafond et les intervalles des colonnes qui se soutiennent sont exactement remplis dans l'intérieur par les tableaux d'une curiosité qui cherche à se satisfaire $[. . .]^{4}$.

1 Cette topique très remarquable et remarquée des plaisirs sensoriels dans les contes de fées croise alors les préoccupations des philosophes sensualistes, surtout à partir des années 1740-1750, une fois qu'un certain consensus s'est établi avec Deslandes, La Mettrie, Condillac et Diderot. L'exemple qui vient immédiatement à l'esprit est le conte «sensualiste » de ce dernier, L'Oiseau blanc, conte bleu $\left(1749^{5}\right)$ avec sa combinaison plus inédite du toucher et de l'ouie, conformément à la nouvelle valorisation du toucher comme sens décisif entre tous, celui qui permet de se connaitre dans le monde. Jean Starobinski ${ }^{6}$ a ainsi montré comment l'excitation simultanée d'un double registre sensoriel (raconter, chatouiller) dans le conte de Diderot était un prélude ou un écho aux deux Lettres (sur les aveugles à l'usage de ceux qui voient, contemporaine du conte, publiée en 1749 , et sur les sourds et muets à l'usage de ceux qui entendent et qui parlent, publiée en 1751) et définissait dans toute son œuvre une poétique de "l'écouter-jouir ». Outre cette analogie sensorielle, l'expérience - car c'en est une - explore aussi toute la gamme graduelle des sensations, de l'effleurement au "toucher dur", moment de bascule où l'extrême plaisir court le danger de se retourner en extrême douleur ${ }^{7}$. Ou, pour le dire autrement: bascule où l'augmentation sensorielle et même ici polysensorielle peut s'inverser en mutilation et la jouissance surhumaine se retourner en inquiétante monstruosités. C'est ce point de l'extrême sensibilité ou sensorialité dans quelques contes postérieurs aux années 1740, au moment où s'est donc établi un consensus sur le primat du sensible, auquel je souhaite m'attacher, afin de montrer comment le conte de cette époque, en recyclant des motifs folkloriques aussi traditionnels que celui des "doués", se fait une nouvelle fois laboratoire expérimental'. Au lieu d'une augmentation sensorielle hérö̈sante ayant une fonction narrative directe, les contes d'une large seconde moitié du XVIII siècle, lorsqu'ils font intervenir des héros "augmentés ", expérimentent plus volontiers les dérèglements sensoriels : il ne s'agit plus dès lors de mettre en avant les harmonies polysensorielles et les exploits, mais les dysharmonies et les catastrophes, et plutôt que de « talents » on parle alors de «manies ». Les héros d'ailleurs ne sont pas toujours « augmentés » mais peuvent être diminués ou dés-organisés, au sens figuré comme au propre, sans organe sensoriel ou aux organes sensoriels déplacés. Ainsi, le conte féerique, en s'hybridant d'un discours médical et anatomique, se fait épreuve des sens, exploration des infinies possibilités entre-aperçues lors de cas de privation, augmentation ou combinaison sensorielles. 


\section{Les doués et autres héros augmentés}

Le héros augmenté dans les contes apparaît d'abord au travers du motif folklorique des "doués", répertorié sous la catégorie des "personnages doués de pouvoirs extraordinaires ${ }^{10} »$. Parmi les cinq sous-catégories établies par Stith Thompson, deux nous intéressent particulièrement: d'abord, celle des "compagnons extraordinaires " (F 600), qui sont des personnages intervenant en groupe, dotés le plus souvent d'une force extraordinaire mais aussi, pour certains d'entre eux, d'un don qui touche les sens. Secondement, c'est aussi la sous-catégorie de ceux qui ont des "pouvoirs extraordinaires de perception " (F 640) qui requiert notre attention ${ }^{11}$. C'est ce motif qu'on trouve dans le conte-type (répertorié par les folkloristes français, Paul Delarue et Marie-Louise Tenèze sous le $\mathrm{n}^{\circ} 513^{12}$ ) «le bateau qui allait sur terre comme sur mer ", plus connu sous l'appellation des (six) compagnons : le héros, dénué lui-même de don si ce n'est celui d'une intelligence ou d'une habileté remarquables, parvient à ses fins grâce à l'aide de cinq compagnons, aux sens ou organes augmentés. Les deux augmentations sensorielles les plus communes étant une vue extraordinairement perçante et une ouie incroyablement fine, comme chez Basile, où, dans le conte "L'ignorant ", un personnage ${ }^{13}$ nommé " Oreilles-de-lièvre » colle son oreille au sol et entend tout ce qui se dit dans le monde entier. Chez Grimm, dans la version «Six à qui rien ne résiste ", les compagnons sont composés d'un tireur à l'arc à la vue perçante, qui peut toucher l'œil gauche d'une mouche à dix lieues, d'un homme doué d'une force extraordinaire, d'un autre d'un souffle puissant, d'un coureur rapide et d'un dernier au don surprenant et difficile à définir, celui de produire un froid glaçant à partir de l'oreille... Dernier exemple : dans un autre conte de Basile, "La Puce », sont mis en scène sept frères chargés de sauver leur sœur Porziella prisonnière d'un ogre et l'on retrouve en Mase, la «fine-oreille » et en Ceccone, la vue perçante. Mais ce conte est également intéressant parce qu'il recourt à une autre augmentation sensorielle traditionnelle, celle de l'odorat puissant de l'ogre : en effet, un roi élève une puce qui grossit jusqu'à atteindre la taille d'un agneau, puis il l'écorche et promet sa fille Porziella à celui qui identifierait, à partir de sa seule peau, l'animal monstrueux. Après plusieurs tentatives vaines de prétendants divers, arrive enfin un ogre qui, grâce à un odorat particulièrement développé, reconnaît l'animal et emporte la belle. On pense alors bien entendu à Perrault où l'augmentation de l'odorat semble de surcroît se combiner avec celle du goût, l'ogre du «Petit Poucet " «fleurant " «à droite et à gauche, disant qu'il sentait la chair fraîche $»^{14}$. Bref, on le voit, les augmentations sensorielles $\mathrm{du}$ folklore, principalement concentrées sur l'ouïe et la vue, sont relativement simples (même quand elles sont inventives et associées à d'autres dons étonnants), dirigées exclusivement vers la résolution du méfait et caractérisées par l'exploit. Rien de tel dans un conte de Cazotte, inséré dans La Suite des Mille et Une Nuits publiée en 1788, pourtant assez proche du conte-type et qui reprend clairement le motif des doués, «Les Prouesses et la mort du capitaine Tranchemont ${ }^{15}$ ».

Dans ce conte, les dons, de fait, ne « servent » à rien, ils n'ont pas vraiment de fonction narrative, ne convergent pas vers une grande action finale, un exploit militaire. Le titre, de tradition romanesque et burlesque (celle des romans comiques ou picaresques et d'une veine rabelaisienne) bien plus que merveilleuse, est un leurre. Le récit, mené sous la forme d'une réduplication d'épisodes guerriers sans véritable trame directrice, présente bien à la fin une série d'exploits mais inutiles, gratuits, inefficaces et s'achève par la mort du héros au profit de son ennemi. Ainsi aucun des nombreux talents dont le 
conte est prolixe ne permet la victoire ni la défaite des armées : la victoire des ennemis vient, au contraire, non pas d'une augmentation mais d'une privation sensorielle, celle de l'ouie. De fait, le talent d'un des "doués", Toujoursdort, celui de faire un bruit extraordinaire, n'a aucun effet, puisque les soldats ennemis, se privant de l'ouïe grâce à des tampons de coton mis dans les oreilles, l'emportent ${ }^{16}$.

Il s'agit bien pourtant d'une reprise du thème folklorique des compagnons «doués ». Tranchemont, caractérisé par une force extraordinaire, s'entoure ainsi de pas moins d'une dizaine de compagnons aux dons variés : le classique et bien nommé Percevue, le chasseur-tireur Droitaubut, le coureur Fendl'air, le derviche Prétaboire, un autre héros de la force, Bondos, mais aussi des personnages aux talents, lorsqu'ils sont traditionnels, plus ou moins redondants ou bizarres qui explorent justement des possibilités organiques ou sensorielles nouvelles. Outre les « forts » déjà évoqués, on en trouve plusieurs autres, Brasdefer et Dentd'acier, mais aussi deux souffleurs, Soufflefeu qui, en attisant le feu, brûle tout et Grossitout qui souffle par exemple dans sa bourse pour la transformer en une immense tente. Le récit compte aussi un ronfleur Toujoursdort déjà évoqué et un faiseur de pluie Grippenuage. Par ailleurs, les talents ne sont pas uniquement du côté des "compagnons ", l'armée ennemie étant également dirigée par un « doué » à la force extraordinaire, Bigstaf. En outre, la multiplication des doués va de pair avec une grande complexification des talents. Toujoursdort, le ronfleur, semble avoir un "talent médiocre » mais celui-ci, si besoin, se diversifie et s'intensifie : il peut faire un bruit effroyable en se tapant le ventre, en se frottant les doigts et en poussant des hurlements, à tel point qu'il a, dans le conte, un double nom et est aussi appelé le Tambour ${ }^{17}$. Surtout, les dons n'ont d'effet ni d'efficacité que par leur combinaison, elle-même tout entière dirigée vers une polysensorialité. Ainsi, les dons, même quand ils ne sont apparemment pas directement liés à une augmentation sensorielle, convergent néanmoins vers un seul but: le plaisir sensoriel et tout particulièrement celui du goût. À plus d'un titre le conte peut, de fait, être lu comme un conte sur la nourriture, le plaisir de la table : non seulement le récit abonde en aliments et en talents qui ont trait à l'alimentation (manger et boire en excès), mais les autres talents sont également le plus souvent mis au service des repas. Bondos, par exemple, fait la preuve de sa force en portant trois énormes choux, Percevue, Fendl'air et Soufflefeu combinent leurs talents pour se procurer gibier et volaille, Soufflefeu dirige son talent du côté de la rôtisserie : "Il fait cuire des cailles en l'air, pour qu'il lui en pleuve dans sa bouche de toutes rôties ${ }^{18}$ ! "Tranchemont, lui-même, casse des pierres d'un coup de sabre, les broie et les mange - allusion à la statue pilonnée et ingérée au début du Rêve de D'Alembert de Diderot ? L'organe exceptionnel («c'est un morceau de dure digestion pour d'autres estomacs que les nôtres $\left.{ }^{19} »\right)$ prélude à une sensorialité extraordinaire. Et de fait, la trame folklorique traditionnelle tendue vers l'exploit guerrier est ici redirigée... vers le plaisir exclusif de la bouche ${ }^{20}$. Avant de livrer bataille, Tranchemont s'adresse de la sorte à sa troupe : « Mais allons souper. - c'est le meilleur parti à prendre à présent [... ${ }^{21}$. » D'ailleurs, dans une tonalité beaucoup plus sinistre, la violence sous-jacente de l'obsession ogresse du manger chez le héros est exhibée par Bigstaf qui traite Tranchemont non de guerrier, mais de "boucher de profession ${ }^{22}$ ». On peut alors considérer qu'une même pulsion de jouissance gustative anime la guerre ou le repas festif (tous deux des "arts de la bouche») qui sert d'épreuve qualifiante des héros et de thème obsédant tout au long du conte. C'est en effet cette puissance sensorielle extrême du goût qui est la vraie quête de ce récit. Les talents ne servent qu’à 
cela : célébrer le plaisir de la bouche. Une fois le veau et les choux recueillis, la force de Tranchemont est utilisée pour fabriquer des plats :

Mon général, lui dit-il [c'est le derviche qui parle], c'est à vous à montrer à présent à ces braves gens ce que vous savez faire. Voilà le veau à la broche, et les choux coupés en morceaux ; mais nous n'avons rien pour recueillir le jus du rôti, point de plat pour assaisonner les choux, levez adroitement sur toute sa longueur une tranche du biscuit qui est devant ma porte et procurez-nous ainsi les vaisseaux nécessaires pour contenir notre sauce et nos légumes ${ }^{23}$.

Alors, le capitaine tire son sabre, "enlève une feuille d'un demi pouce sur toute la longueur du banc de pierre, il y pratique un réservoir pour le jus du rôti, et la feuille qu'il a enlevée sert de plat pour les choux ${ }^{24} »$. La scène se prolonge encore sur plusieurs pages, Bondos fait office de tournebroche, Soufflefeu attise le feu. Et les scènes de ce type se multiplient, dans l'inventivité jouissive d'un détournement du motif des doués : Percevue traque le gibier, Droitaubut bande son arc et le tue à "dix lieues de là ", Fendl'air le ramasse, Bondos le dépouille et le met en broche, Grippenuage attrape un nuage et le crève pour servir de boisson ou pour « rafraîchir l'air » en répandant " une petite rosée $\aleph^{25}$, Fendl'air, encore lui, rapporte des liqueurs volées ou des figues d'Afrique, tandis que Toujoursdort, une fois le ventre tendu par la satisfaction du repas, «imite le tambourin en frappant sur ses joues ${ }^{26}$ ». Le festin est bien ainsi l'occasion d'une polysensorialité étendue : c'est aux moyens de la vue et de l'ouïe que la fête combinée de l'odorat, du goût et encore de l'ouïe (la musique) est rendue possible. Quant au toucher, il est partout: dans les gestes mobilisés par la force extraordinaire, mais aussi dans ceux de la cuisine auxquels participent chacun des doués, dans les "doux oreillers » disposés pour les convives, et même dans la caresse délicate de la rosée. La polysensorialité permet ainsi l'exploration imaginaire d'une synesthésie où les sensations ne seraient plus discontinues mais simultanées, produisant non une addition de plaisirs mais un plaisir synthétique, unique, inconnu, né de la correspondance analogique de plusieurs sens.

On se rappelle alors le début célèbre de l'article « Jouissance » de Diderot : « On possède souvent sans jouir. À qui sont ces magnifiques palais? Qui est-ce qui a planté ces jardins immenses? C'est le souverain : qui est-ce qui en jouit? c'est moi ${ }^{27}$. » Chez ces nouveaux " doués ", le talent ne sert à rien, ne sert même personne, ces "doués » ne sont plus des puissants, mais des jouisseurs: ils jouissent d'eux-mêmes, leur sensorialité exceptionnelle n'est plus un talent mais une pulsion - essentiellement auto-érotique.

\section{Le dérèglement sensoriel}

Une première expérimentation de dérèglement sensoriel est présente dès 1735 dans un conte de Catherine de Lintot, Tendrebrun et Constance ${ }^{28}$, qui met en scène un roi, l'Indolent, né sans tête et "réparé " par une tête postiche, articulée au moyen de ressorts. Pour suppléer à l'absence de la plupart des organes sensoriels, l'œil pour la vue, l'oreille pour l'ouie, la bouche pour le goût et le nez pour l'odorat, une fée redistribue ces organes dans d'autres points du corps : «[...] une bouche à la fossette ${ }^{29}$ $\mathrm{du}$ cou, une oreille dans la main gauche, et un œil dans la droite ${ }^{30}$. " Ainsi, Indolent, pour voir l'héroïne Constance qu'il désire pour femme ${ }^{31}$, met sa main «tout auprès de son visage ${ }^{32}$ ». Quant au nez, il n'est pas replacé, parce que «c'était, selon la fée, une chose inutile, ainsi il ne sent[ait] rien ${ }^{33} »$. Cette absence d'odorat s'étend alors, grâce à 
la polysémie du verbe, à son être tout entier: l'Indolent, conformément à son étymologie, est un insensible. Enfin, cette insensibilité ne relève pas du seul domaine du sentiment amoureux mais de tout sentiment et sens moral : la désorganisation et privation sensorielles (évidemment étendues dans ce conte largement moralisateur où s'opposent Vices et Vertus au « sens » du jugement dont le cerveau, « la tête », serait le siège) provoquent en effet chaos politique et règne de l'injustice. Pour le dire autrement: dans ce conte, le dés-organique désorganise car la sensorialité est le fondement même de toute société.

5 L'un des épisodes d'un autre conte expérimente également le cas d'un dérèglement sensoriel lié encore une fois à un manque physiologique, ayant des incidences directes et extravagantes sur la sensibilité. Dans La Princesse Minon-Minette et le Prince Souci ${ }^{34}$, publié en 1741 et attribué à Caylus, deux personnages, Diafane et Fluet, aux noms programmatiques, se caractérisent par une privation physique : ils sont dépourvus de " poids ", " d'épaisseur ", trop légers ou trop minces, ils sont inadaptés au monde réel. C'est le portrait de Diafane qui est le plus développé : à partir d'une syllepse, le sens figuré d'« être légère » étant pris au sens propre, le récit raconte comment la princesse s'envole au moindre souffle et file notamment une scène cocasse où un prince court après la princesse à travers la campagne tandis qu'elle s'élève et s'échappe de plus en plus vite, poussée par un vent de plus en plus fort, tel un ballon ${ }^{35}$. Des outils compensatoires sont alors nécessaires pour la maintenir coûte que coûte dans le monde réel et terrestre et empêcher qu'elle ne séjourne dans les sphères subtiles, comme un cordon pour la rattacher au sol. Est-elle alors « augmentée " par des sens plus déliés ou "diminuée " par un manque de pesanteur? En tous cas, la bizarrerie sensorielle, dans les deux contes cités, est au cœur d'une interrogation sur le pouvoir décisif des sens dans la société.

Mais l'expérimentation contraire existe aussi : que se passe-t-il cette fois-ci lorsque l'individu n'est pas un insensible mais un hypersensible? Bien entendu, l'expérience est " genrée » : si l'insensible était un homme, l'hypersensible est une femme ${ }^{36}$, qu'on rencontre dans un conte anonyme de 1743 sous le nom transparent, quoique fade au regard des dérèglements de l'héroïne, de Princesse Sensible. Pourtant, l'argument du conte un temps attribué à Marguerite de Lubert, La Princesse Sensible et le Prince Typhon ${ }^{37}$, est particulièrement rebattu et mince : deux héros dotés par deux fées de caractères antagonistes finissent par s'épouser. Ainsi, la princesse Sensible est douée d'une délicatesse maladive et vit dans une Cour précieuse, tandis que le prince Typhon est un géant à la fois rustique et suffisant. Le récit raconte l'éducation du prince qui se défait de son amour-propre et de sa rugosité et, in extremis, les efforts de la princesse pour maîtriser son hypersensibilité, qui permettent bien sûr le mariage final. C'est néanmoins précisément cette "minceur » de l'intrigue, sa linéarité et la quasi-absence de tout merveilleux qui contribuent à en faire un conte de choix pour notre sujet, pour peu qu'on le relise à nouveaux frais. De fait, aucun objet magique ni intervention merveilleuse ne déclenchent la résolution finale du conte. Le seul objet à caractère vaguement merveilleux, appartenant au secteur du merveilleux scientifique, est un temple optique qui projette les images vaniteuses de ceux qui y pénètrent et sert d'outil d'analyse morale, à la manière des inventions catoptriques du conte de Deslandes, L'Optique des mœurs, opposée à l'optique des couleurs, paru juste avant, en $1741^{38}$, et qui sert sans doute de modèle ici. C'est la "maladie» polysensorielle de la bien-nommée princesse Sensible qui nous intéresse ici. Il s'agit en fait d'une qualité 
« supplémentaire » (en plus de la beauté et de l'esprit) conférée à l'héroïne par la fée Prudalie lors d'une scène topique " des dons »:

Dans l'instant que la princesse vint au monde, Prudalie la doua: une figure charmante, un esprit sublime furent les présents de la fée, mais suivant son goût elle crut ne pouvoir ajouter un trop grand degré de délicatesse à deux dons si précieux; pour y parvenir, elle multiplia les tours de baguette et les paroles nécessaires. Sensible, c'est le nom qu'elle lui donna, s'en trouva accablée ${ }^{39}$.

"Accablée»: la qualité de sensibilité se retourne immédiatement en handicap sensoriel, ici, non par privation sensorielle mais bien par augmentation, par hypersensorialité. L'hypersensibilité est de fait, dans ce conte "sensualiste", étroitement liée à l'hyper-sensorialité, exprimée en des termes qui concernent au moins quatre des cinq sens de la princesse : ouïe fine, odorat développé, toucher délicat et vue sensible.

[...] des fibres aussi fins et aussi déliés que les siens, affaiblis encore par une éducation trop complaisante, lui rendaient l'impression des sens d'une délicatesse très embarrassante. Un bruit léger qui la surprenait, même pendant le jour, lui causait une révolution toujours dangereuse, l'odeur des fleurs les plus simples lui donnait des maux de tête insupportables, le velours et le satin étaient rudes à son toucher et ses beaux yeux, loin de pouvoir soutenir l'éclat du soleil, ne pouvaient regarder longtemps sans éblouissement les couleurs un peu vives ${ }^{40}$.

Remarquons d'emblée que le conte pose les termes du «méfait » initial, embrayeur narratif, en langage anatomico-médical (les fibres). Rappelons que pour les théoriciens sensualistes, et notamment les vitalistes de Montpellier, la « fibre » animale est le cœur et l'origine de la sensibilité qui définit l'animal (dont fait partie l'humain). C'est la ligne exposée dans l'article "Sensibilité » de l'Encyclopédie de Diderot et d'Alembert, rédigé par le médecin de Montpellier, Fouquet, qui cite également abondamment Bordeu, l'ami de Diderot et le personnage du Rêve de d'Alembert : il y a une "sensibilité générale des parties » et les organes sont conçus comme des «centres de sensibilité », les nerfs formant «la base et l'essence de tous les organes » et ce "premier et unique nerf » central propre à chaque organe se prolongeant en des fibres qui en sont les « rejetons ». Dans cette théorie, l'excitation violente des fibres (Fouquet dans l'Encyclopédie parle "d'irritabilité » des nerfs, de "fièvre nerveuse ", le Bordeu du Rêve, "d'éréthisme ») produit précisément une hypersensibilité pathologique. On touche alors, dans le cas de ce qu'on peut donc appeler les symptômes de la princesse Sensible, à l'expression d'une " manie ${ }^{41}$ ", maladie marquée justement non par une augmentation sensorielle, à la manière des talents des doués, mais par une diminution ${ }^{42}$ : la vue de la princesse maniaque n'est pas perçante, au contraire, du fait de son excessive irritabilité, elle blesse et n'est donc pas augmentée mais bien diminuée. Le paradoxe, en effet, est que l'augmentation sensorielle s'accompagne ici d'une diminution de la capacité sensorielle. À la grande différence des "doués ", le développement d'un sens chez les hypersensibles pose des problèmes d'adaptation sociale, ou alors il faut qu'il soit à l'échelle même d'un peuple tout entier : les habitants de l'île du Repos dans "Étoilette ${ }^{43}$ " sont ainsi dotés d'une hypersensibilité auditive, ils ne supportent pas le bruit, parlent «toujours bas", marchent "sur la pointe du pied». Cette expérience sensorielle devient alors un modèle heuristique pour imaginer une utopie bienheureuse: sans bruit, point d'éclat violent, "jamais de querelles, rarement des guerres; et quand il fallait absolument en soutenir une, les dames seulement combattaient de loin à coup de pommes d'api ${ }^{44} »$.

7 On le voit, dans le domaine des sens, comme dans bien d'autres, le conte du XVIII ${ }^{e}$ siècle et tout particulièrement cette seconde ou troisième génération de contes d'après les 
années 1740 , est un laboratoire expérimental. Selon un protocole préétabli (comme modifier par exemple une capacité sensorielle, par diminution, augmentation, privation voire déplacement de l'organe percepteur, ou encore combiner une ou plusieurs de ces modifications), on observe ainsi le déroulement de l'expérience et on constate son résultat. L'expérience-y compris celles menées à la paillasse - est épreuve, on le sait, jamais preuve ${ }^{45}$ et les notions de fiction (scientifique) et d'hypothèse ne sont pas clairement distinctes au $\mathrm{XVIII}^{\mathrm{e}}$ siècle $^{46}$. Après tout, de façon évidemment plus brouillonne et moins systématique, les contes font ou refont (selon leur date de publication), à leur manière "excessive » de lentille grossissante, l'expérience heuristique de la statue dans le Traité des sensations de Condillac en 1754, c'est-à-dire ouvrir au monde un sens oblitéré jusque-là, borner d'abord l'individu à ce seul sens, puis en ouvrir plusieurs, les combiner, à deux, à trois, tous, etc. Quelle(s) conclusion(s) en tirer dans les quelques contes explorés ici ? Déjà, la même que celle, décisive, du sensualiste : je suis mes sensations, respirant seulement l'odeur de rose, je suis la rose ${ }^{47}$. Mais bien d'autres encore, aussi diverses que le sont les expériences menées: augmentation et saturation sensorielles peuvent provoquer des plaisirs extrêmes, d'une violence telle qu'ils peuvent s'inverser en souffrance tout aussi extrême, voire fatale. Car on peut mourir de plaisir - et à ce titre, le sens sans doute, dans nos contes, le plus naturellement sensible et potentiellement susceptible de provoquer querelles, guerres, destructions et morts, est l'ouie. Diderot l'évoque dans ses réponses aux objections sur sa Lettre sur les sourds et muets de 1751 :

En musique, le plaisir de la sensation dépend d'une disposition particulière non seulement de l'oreille mais de tout le système des nerfs. S'il y a des têtes sonnantes, il y a aussi des corps que j'appellerais volontiers harmoniques ; des hommes, en qui toutes les fibres oscillent avec tant de promptitude et de vivacité, que sur l'expérience des mouvements violents que l'harmonie leur cause, ils sentent la possibilité de mouvements plus violents encore, et atteignant à l'idée d'une sorte de musique qui les ferait mourir de plaisir. Alors leur existence leur paraît comme attachée à une seule fibre tendue, qu'une vibration trop forte peut rompre ${ }^{48}$.

D'où l'autre conclusion possible également qui en découle : le sadisme, la "boucherie ", font aussi partie des plaisirs - éventuellement mortels mais suprêmes. Et encore : le fondement de notre société policée (celle du XVIII siècle mais aussi la nôtre ?) tient à la modération et à l'équilibre sensitifs. Et enfin : la combinaison analogique des sens, leurs correspondances, laissent percevoir une vision décentrée et élargie du monde sensible et, à l'horizon, la possibilité d'un plaisir non pas violent mais sublime, un plaisir de rapports. Et c'est toujours Diderot qui le dit: les morceaux de musique «qui affectent agréablement sans réveiller en vous ni peinture, ni perception distincte de rapports, ne flattent votre oreille que comme l'arc-en-ciel plaît à vos yeux, d'un plaisir de sensation pure et simple; et qu'il s'en faut beaucoup qu'ils aient toute la perfection que vous en pourriez exiger $[. . .]^{49} »$. Dans cette perspective, on peut peut-être considérer que les expérimentations sensorielles des contes sont aussi des outils de perception enrichie, ouvrant les sens du lecteur à des sensations inouïes et à des synesthésies indicibles mais pas ineffables. 


\section{NOTES}

1. "Je suis toute hors de moi, leur disait-elle; je croirais mourir, si je n'étais sûre de mon immortalité. C'en est trop ; laissez-moi : je succombe sous la violence des plaisirs. » Montesquieu, Lettres persanes, Lettre 141, Paris, Le Livre de Poche, 2005, p. 433.

2. Voir dans ce volume l'étude de M. Traoré, «Le conte Ibrahim et Anaïs : le merveilleux comme expérience du droit des femmes au plaisir dans les Lettres persanes (1721) de Montesquieu ».

3. Anonyme, Le Navire volant. Nouveaux Contes de fées, dans Le Cabinet des fées, Amsterdam, et se trouve à Paris, Rue et Hôtel Serpente, 1786 [dans Nouveau recueil de contes de fées, P.-J. Mariette, 1731], t. 31, p. 216-217.

4. Anonyme, Le Prince Ananas et la Princesse Moustelle, dans Le Cabinet des Fées, Genève, Barde, Manget et $\mathrm{C}^{\mathrm{ie}}$, Paris, Cuchet, 1786 [La Haye, 1750], t. 34, p. 401.

5. Date établie définitivement, voir A. Defrance, "Introduction à Diderot ", L'Oiseau blanc, conte bleu, dans Hamilton, Rousseau Pajon, Cazotte, Tessin, Duclos, Diderot, Contes, éd. A. Defrance et J.Fr. Perrin, Paris, H. Champion, coll. « Bibliothèque des Génies et des Fées », n 16, 2008, p. 1416.

6. J. Starobinski, «Du pied de la favorite au genou de Jacques», dans Denis Diderot, 1713-1784. Colloque international Paris - Sèvres - Rems - Langres (4-11 juillet 1984), Actes recueillis par A.M. Chouillet, Paris, Aux amateurs de livres, 1985, p. 359-380.

7. «Mademoiselle, vous avez ce soir le toucher dur et vous me chatouillez trop fort. Doucement, doucement... Fort bien, comme cela... [...]. » D. Diderot, L'Oiseau blanc, conte bleu, éd. citée, p. 1446. Voir aussi A. Gaillard, «Contage et sexualité : le récit à double entente de Diderot, L'Oiseau blanc, conte bleu », dans A. Defrance et J .--Fr. Perrin (dir.), Le conte en ses paroles. La figuration de l'oralité dans le conte merveilleux du Classicisme aux Lumières, Paris, Desjonquères, coll. "L'esprit des lettres », 2007, p. 200-215.

8. Je rejoins l'hypothèse stimulante d'E. Sempère (à propos de Cazotte, Lubert ou Lamekis de Mouhy) sur la corrélation entre désordre des sensations, primat du sensible et de la sensorialité, et suspens de l'interprétation symbolique comme l'un des moteurs du fantastique. Voir «Le merveilleux à l'épreuve des sens : une phénoménologie sous contraintes (fin $\mathrm{XVII}^{\mathrm{e}}$ siècle-XVIII ${ }^{\mathrm{e}}$ siècle) ", Féeries, n 14, 2017, mis en ligne le 31 juillet 2017, consulté le 13 février 2018. Disponible sur http://journals.openedition.org/feeries/1174

9. Voir R. Jomand-Baudry et J.-Fr. Perrin (dir.), Le Conte merveilleux au XVIII ${ }^{e}$ siècle : une poétique expérimentale, Paris, Kimé, 2002.

10. ${ }^{\mathrm{F}}$ 600-F 699: "Persons with extraordinary powers ", dans S. Thompson, Motif-index of folkliterature: a classification of narrative elements in folktales [...], Bloomington, Indiana University Press, 1955-1958. Disponible sur http://www.ruthenia.ru/folklore/thompson/.

11. Ce qui correspond dans le premier ouvrage des folkloristes anglo-saxons Aarne et Thompson (The Types of the Folktale, 1928) aux contes-types 513 A « six Go through the whole world » et 513 B "The Land and water ship ».

12. J. Bru et B. Bonnemason, Le Conte populaire français. Contes merveilleux. Supplément au Catalogue de Paul Delarue et Marie-Louise Tenèze, Toulouse, PUM, 2017.

13. G. Basile, "L'Ignorant », dans Le Conte des contes ou le Divertissement des petits enfants, trad. Fr. Decroisette, III, 8, Strasbourg, Circé, 1995, p. 275.

14. Ch. Perrault, « Le Petit Poucet », dans Contes, G. Rouger (éd.), Paris, Garnier, 1967, p. 192.

15. J. Cazotte, «Les Prouesses et la mort du capitaine Tranchemont, de ses braves. Conte de Dobil-Hasen ", dans Le Cabinet des fées, La Suite des Mille et une nuits, contes arabes, Genève, Barde, Manget et $\mathrm{C}^{\text {ie }}$ et Paris, Cuchet, 1788, t. 39, p. 421-478 [éd. critique R. Robert, La Suite des Mille et une nuits, Paris, H. Champion, coll. "Bibliothèque des Génies et des Fées », n 10, 2012, p. 490-515]. C'est Raymonde Robert qui, la première, m'a fait découvrir cette histoire insérée, lors du colloque 
«Les êtres merveilleux », A. Defrance et M. Poirson (dir.), Université Stendhal - Grenoble 3, 16-17 octobre 2014. À paraître en 2018 sous le titre Étranges créatures : les êtres merveilleux dans les arts de l'âge classique au postmodernisme, Paris, Éditions Orizons, coll. « Comparaisons ».

16. Ibid., p. 478.

17. Ibid., p. 440.

18. Ibid., p. 439.

19. Ibid., p. 442.

20. La dimension rabelaisienne est ici nette.

21. J. Cazotte, ouvr. cité, p. 453.

22. Ibid., p. 476 (je souligne).

23. Ibid., p. 440-441.

24. Ibid.

25. Ibid., p. 455.

26. Ibid., p. 449.

27. D. Diderot, "Jouissance ", dans Encyclopédie ou Dictionnaire raisonné des sciences, des arts et des métiers de Diderot et D’Alembert.

28. C. Cailleau, comtesse de Lintot, Tendrebrun et Constance ["Troisième conte»], dans Trois Nouveaux Contes des Fées, Paris, Didot, 1735, p. 121-220.

29. Diminutif de « fosse », désigne ici un petit creux.

30. C. Cailleau, ouvr. cité, p. 175.

31. Mais ce « désir » n'a rien dans le texte d'un sentiment amoureux ni même voluptueux, aucune motivation particulière n'est donnée, la seule remarque s'y rapportant dans le conte est qu'il « avait envie d'être marié » (ibid., p. 173).

32. Ibid.

33. Ibid., p. 176.

34. [Caylus], La Princesse Minon-Minette et le Prince Souci, dans Le Cabinet des Fées, Genève, Barde, Manget et $C^{\text {ie }}$, Paris, Cuchet, 1786 [1741], t. 34.

35. Ibid., p. 243-247.

36. «Parmi les hommes, les enfants et après eux les personnes du sexe, sont ceux qui sont le plus éminemment sensibles [...]», article "Sensibilité, sentiment [médecine]», Encyclopédie ou Dictionnaire raisonné des sciences, des arts et des métiers de Diderot et D’Alembert, vol. XV, 1765 (art. de Fouquet).

37. Anonyme, La Princesse Sensible et le Prince Typhon, La Haye, 1743. Sur la question de l'attribution, voir l'introduction d'Aurélie Zygel-Basso qui la récuse, à très juste titre me semblet-il, étant donné l'extrême linéarité du récit et la quasi-absence de tout merveilleux. Dans Mademoiselle de Lubert, Contes, Paris, H. Champion, coll. «Bibliothèque des Génies et des Fées ", $\mathrm{n}^{\circ} 14,2005$, p. 30-31.

38. Voir B. Roukhomovsky, « Des effets merveilleux de l'optique », Féeries, n² 2, 2005, p. 259-272.

39. Anonyme, La Princesse Sensible et le Prince Typhon, ouvr. cité, p. 460.

40. Ibid., p. 461.

41. À la différence de la folie, la manie se définit comme un état de fureur et non d'abattement. «On appelle de ce nom un délire universel sans fièvre, du moins essentielle : assez souvent ce délire est furieux, avec audace, colère, \& alors il mérite plus rigoureusement le nom de manie ; s'il est doux, tranquille, simplement ridicule, on doit plutôt l'appeler folie, imbécillité. » Article « Manie » de Ménuret de Chambaud, Encyclopédie ou Dictionnaire raisonné des sciences, des arts et des métiers de Diderot et D'Alembert, vol. X, 1765.

42. «[...] les yeux sont frappés, éblouis de temps en temps par des traits de lumières, des espèces d'éclairs ; les oreilles sont fatiguées par des bruits [...]. ». Ibid. 
43. L'un des deux contes ajoutés par Marguerite de Lubert dans la réédition qu'elle fait de l'ouvrage de Henriette-Julie de Castelnau de Murat, Les Lutins du château de Kernosy [1710], Leyde, 1753.

44. M. de Lubert, «Étoilette», dans Contes, A. Zygel-Basso (éd.), Paris, H. Champion, coll. «Bibliothèque des Génies et des Fées ", n 14, 2005, p. 378.

45. Voir Br. Latour et S. Woolgar, La vie de laboratoire. La production des faits scientifiques [1979], Paris, La Découverte, 2003.

46. Voir I. Moreau, « La science entre fiction et hypothèse, de Descartes à l'Encyclopédie ", La Lettre clandestine, $\mathrm{n}^{\circ} 16,2008$, p. 347-356 ; voir également Penser l'ordre naturel 1680-1810, A. Paschoud et N. Vuillemin (dir.), Oxford, Voltaire Foundation, 2012, notamment la conclusion d'Adrien Paschoud sur l'interaction fiction, expérience, hypothèse. Ou encore, sur la question de l'administration de la preuve dans le récit d'expérience à l'âge classique, voir Ch. Licoppe, La Formation de la pratique scientifique. Le Discours de l'expérience en France et en Angleterre (1630-1820), Paris, La Découverte, 1996. Enfin, plus orienté sur le récit de fiction expérimental, notre contribution ( $2^{\mathrm{e}}$ part., chap. XI) «Écrire la science à l'époque des Lumières : comment le récit de fiction peut-il faire preuve?», dans J. Ducos (dir.), Les Sciences et le Livre. Formes des écrits scientifiques des débuts de l'imprimé à l'époque moderne, Paris, Hermann, 2017, p. 221-235.

47. É. Bonnot de Condillac, « Elle sera donc odeur de rose, d'œillet, de jasmin, de violette, suivant les objets qui agiront sur son organe", dans Traité des Sensations [1754], Paris, Fayard, coll. «Corpus des œuvres de philosophie en langue française », 1984, p. 15.

48. D. Diderot, "Lettre à Mademoiselle... ", dans Lettre sur les aveugles. Lettre sur les sourds et muets, M. Hobson et S. Harvey (éds), Paris, GF Flammarion, 2000, p. 147.

49. Ibid., p. 146.

\section{RÉSUMÉS}

L'augmentation sensorielle dans les contes apparait d'abord au travers du motif folklorique des « doués ", répertorié sous la catégorie des " personnages doués de pouvoirs extraordinaires ».

$\mathrm{Au} \mathrm{XVIII}^{\mathrm{e}}$ siècle, l'exploration des possibilités ouvertes par les sens, en résonnance avec la philosophie sensualiste, prend néanmoins une toute autre ampleur. Les contes peuvent alors être considérés comme de véritables laboratoires d'expérimentations sensorielles qui mettent en scène toutes sortes de dérèglements des sens, par diminution, augmentation, privation voire déplacement de l'organe percepteur, ou encore combinaison et correspondance de plusieurs sens. L'étude vise alors à examiner dans un corpus de contes, pour l'essentiel de la seconde moitié du XVIII ${ }^{\mathrm{e}}$ siècle, avec des auteurs comme Cazotte ou Lubert, les variations et déplacements que subit le motif traditionnel de l'augmentation sensorielle complètement réinventé à cette occasion.

In tales, sense augmentation first appeared through the folk-literature motif of "the gifted", or "persons with extraordinary powers". In the 18th century, however, the exploration of the possibilities opened by the senses, echoing with sensualist philosophy, gained a whole new dimension. Tales can thus be truly considered as laboratories of sensory experiment displaying all sorts of sensory troubles triggered by the diminution, augmentation or removal of the receiver organ, or by a combination and correspondence of several different senses. The object of 
this study is therefore to determine to what extent the wholly recreated folk-tale motif of sensory increase varies or differs, in a corpus of tales from the second half of the 18th century, with such authors as Cazotte and Lubert.

INDEX

Mots-clés : augmentation sensorielle, polysensorialité, synesthésie, Jacques Cazotte, Marguerite de Lubert

Keywords : sensory augmentation, polysensoriality, synaesthesia, Jacques Cazotte, Marguerite de Lubert

\section{AUTEUR}

AURÉLIA GAILLARD

Université Bordeaux Montaigne, SPH 